\title{
Relationship between adiponectin, TNFa, and SHBG in prepubertal children with obesity
}

\author{
Marta Ramon-Krauel ${ }^{1}$, María Jesús Leal-Witt ${ }^{1}$, Óscar Osorio-Conles ${ }^{1}$, Montse Amat-Bou ${ }^{1}$, Carles Lerin ${ }^{1 *}$ and \\ David M. Selva $2^{*}$
}

\begin{abstract}
Background: Sex hormone-binding globulin (SHBG) levels are low in adult subjects with obesity when compared to normal-weight individuals. Obesity is associated with higher tumor necrosis factor alpha (TNFa) plasma levels and lower adiponectin levels. Moreover, we have recently elucidated the molecular mechanisms by which TNFa and adiponectin regulate hepatic SHBG production.
\end{abstract}

Aim: The main objective of this study was to assess if the adult associations between TNFa, adiponectin, and SHBG are present in prepubertal children.

Methods: We determined several morphometric and biochemical parameters in normal-weight $(n=15)$ and obese prepubertal $(n=51)$ children, as well as quantified plasma SHBG, TNFa receptor 1 (TNFa-R1), and adiponectin levels.

Results: Our results showed that prepubertal children with obesity had decreased plasma SHBG levels compared to normal-weight controls (67 nmol/L vs $172 \mathrm{nmol} / \mathrm{L})$. Importantly, SHBG plasma levels correlated significantly $(P<0.05)$ with TNFa (negatively, Bstd $=-0.31$ ) and adiponectin (positively, Bstd=0.58) suggesting an important role of these two cytokines in determining plasma SHBG levels in prepubertal children.

Conclusions: Our results suggest that plasma adiponectin levels may play a more important role than TNFa in influencing plasma SHBG levels in our prepubertal population with obesity.

Keywords: Prepubertal children, Obesity, Sex hormone-binding globulin, Cytokines

\section{Background}

Childhood obesity prevalence has increased at an alarming rate globally, and the International Association for the Study of Obesity estimates that up to 200 million school-aged children are either overweight or obese [1, 2]. This is important because children and adolescents with obesity are at higher risk of obesity during adulthood [3, 4], which is often linked to serious conditions

\footnotetext{
* Correspondence: clerin@fsjd.org; david.martinez.selva@vhir.org

${ }^{1}$ Endocrinology Department, Institut de Recerca Sant Joan de Déu, Hospital Sant Joan de Déu, 08950 Barcelona, Spain

2Diabetes and Metabolism Research Unit, Vall Hebron Institut de Recerca (VHIR), Universitat Autònoma de Barcelona, Pg Vall d'Hebron 119-129, 08035 Barcelona, Spain
}

\section{Springer Open}

(c) The Author(s). 2021 Open Access This article is licensed under a Creative Commons Attribution 4.0 International License, which permits use, sharing, adaptation, distribution and reproduction in any medium or format, as long as you give appropriate credit to the original author(s) and the source, provide a link to the Creative Commons licence, and indicate if changes were made. The images or other third party material in this article are included in the article's Creative Commons licence, unless indicated otherwise in a credit line to the material. If material is not included in the article's Creative Commons licence and your intended use is not permitted by statutory regulation or exceeds the permitted use, you will need to obtain permission directly from the copyright holder. To view a copy of this licence, visit http://creativecommons.org/licenses/by/4.0/. bolic syndrome, and several types of cancer [3-8].

It is well-known that adult individuals with obesity show lower plasma sex hormone-binding globulin (SHBG) levels than normal-weight subjects $[9,10]$. SHBG is a protein produced and secreted into the circulation by the liver that binds androgens and estrogens with high affinity. In blood, SHBG acts as a carrier of these sex steroids and regulates their bioavailability and access to target tissues and cells [11]. Interestingly, we have previously described that low-grade inflammation occurring in obesity is an important regulator of plasma SHBG levels [12]. Obesity is associated with higher tumor necrosis factor alpha (TNF $\alpha)$ plasma levels and 
lower adiponectin levels in adults $[13,14]$. Moreover, we have recently elucidated the molecular mechanisms by which TNF $\alpha$ and adiponectin regulate hepatic SHBG production [15-17]. Low plasma SHBG levels have been also found in children with obesity; however, the reasons for such reduction need to be yet determined.

In the present work, we aimed to study whether these regulatory mechanisms were already present in prepubertal children. For this purpose, we determined several morphometric and biochemical parameters in normal-weight and prepubertal children with obesity, as well as quantified plasma SHBG, TNF $\alpha$ receptor 1 (TNF $\alpha-R 1)$, and adiponectin levels.

\section{Methods}

\section{Study participants}

The study was approved by the Hospital's Ethic Committee (PIC-21-12). Subsets of data from this cohort have been reported elsewhere [18, 19]. Children with obesity attending the Hospital Sant Joan de Déu, Barcelona (Spain) were recruited between the months of January 2013 and December 2014. Inclusion criteria were children (a) age from 7 to 10 years old; (b) prepubertal, defined as Tanner stage I breast development for girls and testicular volume less than $4 \mathrm{ml}$ in boys; (c) with BMI-SDS for a given age and sex using the World Health Organization (WHO) standards greater than 2 standard deviations for the obesity group, and between -1 and 1 standard deviations for the normal-weight group. Exclusion criteria included any form of endogen obesity, major congenital or chronic disease, druginduced obesity, use of drugs for weight loss, and involvement in another weight-loss program. All parents signed the informed consent document. Blood samples were obtained from the hospital's biobank and were available from 15 normal-weight controls and from 51 of the 53 subjects with obesity initially included in the study.

\section{Physiologic and biochemical measurements}

Blood samples were obtained in the morning after an overnight fast (8-10 h of fasting). We measured weight $(\mathrm{kg})$ and height $(\mathrm{m})$ with light clothing in a calibrated scale and rigid stadiometer. Body mass index (BMI) was calculated, and BMI-SDS was obtained by using the "Anthro Plus" software (WHO). Blood pressure was measured in the right arm using an automated system with the appropriate sleeve size for the arm diameter. Waist circumference was determined as middle point between the last rib and iliac crest. Blood samples were taken in tubes containing EDTA, and plasma was immediately separated, aliquoted, and stored at $-80{ }^{\circ} \mathrm{C}$ until further use. Glucose, insulin, glycated hemoglobin (HbA1c), and the lipid profile (total cholesterol, LDL- cholesterol, HDL-cholesterol, triglycerides) were measured using standard protocols at the Hospital's clinical laboratory. An oral glucose tolerance test (1.75 g glucose $/ \mathrm{kg}$ with a maximum of $75 \mathrm{~g}$ per subject) was performed in a subset of children with obesity $(n=47)$; glucose and insulin levels were measured at 0, 30, 60, 90, and 120 min post-glucose load. The insulinogenic index (IGI) and the whole-body insulin sensitivity index (WBISI) were calculated from the tolerance test data as previously described [20]. Plasma levels for SHBG, adiponectin, and TNF $\alpha-R 1$ were measured by ELISA (R\&D Systems, Madrid, Spain).

\section{Statistical analysis}

Data are shown as mean (SD) for normally distributed data, and median [IQR] for non-normally distributed data; groups were compared using two-paired $t$-test or Mann-Whitney $U$ test, respectively. Normality was tested with the Shapiro-Wilk W test. SHBG levels were log-transformed to achieve normality before testing correlations. Associations between variables were determined by standard least-square regression, with sex, age, and BMI-SDS as covariates where indicated. Data analysis and calculations were performed in JMP v14.3 (SAS Institute Inc., Cary, NC). A $p$ value below 0.05 was considered significant.

\section{Results}

Circulating SHBG levels are decreased in prepubertal children with obesity

The main clinical and biochemical features of normalweight $(n=15)$ and children with obesity $(n=51)$ are displayed in Table 1 . All morphometric parameters were significantly elevated in subjects with obesity compared with lean subjects, including weight, BMI-SDS, and waist circumference (Table 1). Regarding the glycemic parameters, no differences were found between lean and obese subjects in glucose and HbA1c levels; however, insulin concentration and HOMA-IR values were significantly increased in children with obesity (Table 1). Regarding the lipid profile, while total cholesterol was not altered, we observed increased LDL-cholesterol and decreased HDL-cholesterol, as well as higher triacylglyceride (TAG) levels in the group with obesity (Table 1). Moreover, subjects with obesity had significantly higher Creactive protein (CRP) and TNF $\alpha-\mathrm{R} 1$ and reduced SHBG and adiponectin plasma levels compared to the normalweight controls (Table 1 ).

\section{SHBG correlations with anthropometric and biochemical parameters in prepubertal children}

After analyzing all the measured parameters in our study population, our results showed that SHBG levels were negatively correlated to BMI-SDS, waist circumference, 
Table 1 Demographic and physiologic characteristics of study subjects. Normally distributed variables are shown by mean (SD) and groups compared by two-tail t-test. ${ }^{2}$ Non-normally distributed data are shown by median [IQR] and groups compared by MannWhitney $U$ test. Bold font indicates $p<0.0001$. Min, minimum value; Max, maximum value; BMI-SDS, BMI standard deviation score; SP, systolic pressure; $D P$, diastolic pressure; $A D I P O Q$, adiponectin; $T A G$, triacylglycerides; $L D L-C h o$, low-density lipoprotein cholesterol; $H D L$ Cho, high-density lipoprotein cholesterol; n.a., not applicable

\begin{tabular}{|c|c|c|c|c|c|}
\hline & Normal weic & & Obesity $(n=5$ & & \\
\hline & Mean \pm SD & Min-max & Mean \pm SD & Min-max & $p$ value \\
\hline $\operatorname{Sex}(F / M)$ & $7 / 8$ & . & $28 / 23$ & & n.a. \\
\hline Age (years) & $8.4 \pm 0.8$ & $(7.3,10.1)$ & $9.0 \pm 1.1$ & $(7.0,11.0)$ & 0.048 \\
\hline Weight (kg) & $26.8 \pm 2.8$ & $(22.8,34.8)$ & $53.5 \pm 12.6$ & $(32.2,96.3)$ & $<0.001$ \\
\hline BMI-SDS & $-0.01 \pm 0.89$ & $(-1.71,1.3)$ & $3.41 \pm 0.76$ & $(2.04,5.76)$ & $<0.001$ \\
\hline Waist Circ. (cm) & $56 \pm 3$ & $(50,60)$ & $83 \pm 9$ & $(65,104)$ & $<0.001$ \\
\hline $\mathrm{SP}(\mathrm{mmHg})$ & $104 \pm 7$ & $(94,116)$ & $113 \pm 9$ & $(92,143)$ & 0.001 \\
\hline $\mathrm{DP}(\mathrm{mmHg})$ & $64 \pm 6$ & $(52,73)$ & $70 \pm 7$ & $(54,83)$ & 0.003 \\
\hline SHBG $(\mathrm{nmol} / \mathrm{L})^{\mathrm{a}}$ & $172 \pm 24$ & $(115,218)$ & $67 \pm 68$ & $(12,195)$ & $<0.001$ \\
\hline TNFa-R1 (pg/mL) & $854 \pm 230$ & $(578,1435)$ & $1084 \pm 205$ & $(638,1573)$ & 0.001 \\
\hline $\mathrm{CRP}(\mathrm{mg} / \mathrm{L})^{\mathrm{a}}$ & $0.6 \pm 2.1$ & $(0.2,27.4)$ & $3.0 \pm 3.2$ & $(0.5,52.2)$ & 0.005 \\
\hline ADIPOQ $(p g / m L)^{a}$ & $12380 \pm 4739$ & $(6431,20138)$ & $7057 \pm 3778$ & $(1214,17748)$ & $<0.001$ \\
\hline Glycemia (mg/dL) & $86 \pm 6$ & $(77,94)$ & $85 \pm 8$ & $(70,113)$ & 0.765 \\
\hline Insulin (mU/L) & $3.2 \pm 1.6$ & $(0.5,5.7)$ & $13.6 \pm 6.6$ & $(2.1,33.3)$ & $<0.001$ \\
\hline $\mathrm{HbA1c}(\%)$ & $5.3 \pm 0.2$ & $(5,5.7)$ & $5.3 \pm 0.2$ & $(4.9,5.8)$ & 0.894 \\
\hline Cholesterol (mg/dL) & $160 \pm 21$ & $(132,200)$ & $165 \pm 24$ & $(115,214)$ & 0.554 \\
\hline $\operatorname{TAG}(\mathrm{mg} / \mathrm{dL})^{\mathrm{a}}$ & $42 \pm 17$ & $(29,70)$ & $70 \pm 41$ & $(39,183)$ & $<0.001$ \\
\hline LDL-Cho (mg/dL) & $89 \pm 19$ & $(67,132)$ & $104 \pm 23$ & $(63,150)$ & 0.036 \\
\hline $\mathrm{HDL}-\mathrm{Cho}(\mathrm{mg} / \mathrm{dL})$ & $62 \pm 11$ & $(44,82)$ & $44 \pm 10$ & $(29,82)$ & $<0.001$ \\
\hline$|G|^{a}$ & & & $1.27 \pm 1.00$ & $(-0.01,5.69)$ & n.a. \\
\hline WBISI ${ }^{\text {a }}$ & & & $3.06 \pm 2.93$ & $(1.23,11.74)$ & n.a. \\
\hline HOMA-IR & $0.70 \pm 0.35$ & $(0.10,1.25)$ & $2.87 \pm 1.47$ & $(0.44,6.66)$ & $<0.001$ \\
\hline
\end{tabular}

insulin, HOMA-IR, TAG, TNF $\alpha-R 1$, and CRP plasma levels adjusting for sex and age. In addition, plasma SHBG levels correlated significantly and positively with adiponectin and HDL plasma levels (Table 2). No correlation was found between SHBG and glucose, HbA1c, total cholesterol, or LDL levels (Table 2). Interestingly, while further adjustment for BMI-SDS did not substantially modify SHBG correlations with insulin, HOMA-IR, and adiponectin, its association with inflammation markers TNF $\alpha-R 1$ and CRP plasma levels was completely lost (Table 2).

\section{SHBG plasma level correlations in prepubertal children with obesity}

Given that obesity is intimately associated with SHBG levels, insulin resistance, and inflammation markers, we analyzed SHBG correlations with all measured parameters only in prepubertal children with obesity to avoid this confounding factor. Our results showed that SHBG plasma levels correlated significantly and negatively with waist circumference, insulin, and TAG concentration, while they correlated positively with adiponectin (Table $3)$. No correlation was found between SHBG and BMISDS, \% fat mass, TNF $\alpha-R 1$, CRP glucose, total cholesterol, LDL, or HDL plasma levels (Table 3). Further adjustment by BMI-SDS did not substantially modify these correlations (Table 3).

\section{Discussion}

Childhood obesity has become a major health concern in the last decades and has increased its prevalence worldwide, in both developed and developing countries [21-23]. It has been already described that childhood and adolescent obesity is associated with an increase in BMI and excess of adiposity [5, 24] which was also evident in our study since prepubertal children with obesity showed higher BMI-SDS and waist circumference when compared with lean children. This adiposity increase occurring in obesity is also associated with insulin resistance, hypertension, and dyslipidemia, which in turn increase risk of developing atherosclerotic vascular disease and type 2 diabetes [5, 24]. Our results 
Table 2 Correlations between SHBG levels and anthropometric and physiologic parameters in all subjects. Multivariate linear regression was applied to determine correlations between SHBG levels and other variables in all subjects. Bold font indicates $P<$ 0.05. Model 1 adjusted for sex and age. Model 2 adjusted for sex, age, and BMI-SDS. Bstd, standardized beta coefficient from the regression model; BM-SDS, BMI standard deviation score; ADIPOQ, adiponectin; TAG, triacylglycerides; $L D L-C h o$, low-density lipoprotein cholesterol; HDL-Cho, high-density lipoprotein cholesterol; n.a., not applicable

\begin{tabular}{lllll}
\hline & Model 1 & Model 2 & & \\
\cline { 3 - 5 } & Bstd & $\boldsymbol{p}$ value & Bstd & $\boldsymbol{p}$ value \\
\hline BMI-SDS & $-\mathbf{0 . 6 5}$ & $<\mathbf{0 . 0 0 1}$ & - & - \\
Waist circ. & $-\mathbf{0 . 6 1}$ & $<\mathbf{0 . 0 0 1}$ & $-\mathbf{0 . 1 4}$ & $\mathbf{0 . 0 1 3}$ \\
TNFa-R1 & $-\mathbf{0 . 3 1}$ & $\mathbf{0 . 0 1 2}$ & -0.02 & 0.914 \\
log CRP & $-\mathbf{0 . 3 1}$ & $\mathbf{0 . 0 2 2}$ & -0.11 & 0.478 \\
log ADIPOQ & $\mathbf{0 . 5 8}$ & $<\mathbf{0 . 0 0 1}$ & $\mathbf{0 . 5 7}$ & $<\mathbf{0 . 0 0 1}$ \\
Glucose & -0.12 & 0.355 & -0.23 & 0.173 \\
Insulin & $-\mathbf{0 . 7 3}$ & $<\mathbf{0 . 0 0 1}$ & $-\mathbf{0 . 6 3}$ & $<\mathbf{0 . 0 0 1}$ \\
HbA1C & -0.01 & 0.923 & -0.02 & 0.910 \\
HOMA-IR & $-\mathbf{0 . 7 2}$ & $<\mathbf{0 . 0 0 1}$ & $-\mathbf{0 . 6 3}$ & $<\mathbf{0 . 0 0 1}$ \\
TAG & $-\mathbf{0 . 4 8}$ & $<\mathbf{0 . 0 0 1}$ & $-\mathbf{0 . 3 7}$ & $\mathbf{0 . 0 1 8}$ \\
Cholesterol & 0.18 & 0.201 & 0.18 & 0.301 \\
LDL-Cho & 0.09 & 0.516 & 0.21 & 0.225 \\
HDL-Cho & $\mathbf{0 . 4 3}$ & $\mathbf{0 . 0 0 1}$ & 0.16 & 0.269 \\
\hline
\end{tabular}

corroborated the latter since obese prepubertal children had higher insulin plasma levels together with an increase in HOMA-IR and higher LDL and TAG plasma levels when compared with lean prepubertal children.

An excess of adiposity may also influence various aspects of pubertal development, such as timing of pubertal initiation and hormonal parameters during puberty. How obesity may perturb several hormonal aspects of puberty is currently under debate; however, it may involve sex hormone-binding globulin (SHBG), a liverproduced glycoprotein that binds sex steroids with high affinity in blood regulating their bioavailability [11]. Interestingly, SHBG plasma levels change through human life-span, remaining high during infancy and decreasing when puberty approaches [25]. It is important to mention that low plasma SHBG levels are found in children with obesity; this is in agreement with our results showing that prepubertal children with obesity had half the plasma SHBG levels present in normal-weight prepubertal children.

Low plasma SHBG levels are also present in adult subjects with obesity $[9,10]$. The reason why this may occur has been investigated during the last three decades; initially, it was suggested that hyperinsulinemia was the main factor reducing plasma SHBG levels [26, 27]. In fact, a negative correlation between plasma SHBG and
Table 3 Correlations between SHBG levels and anthropometric and physiologic parameters in subjects with obesity. Multivariate linear regression was applied to determine correlations between SHBG levels and other variables only in subjects with obesity. Bold font indicates $P<0.05$. Model 1 adjusted for sex and age. Model 2 adjusted for sex, age, and BMI-SDS. Bstd, standardized beta coefficient from the regression model; BMI-SDS, BMI standard deviation score; ADIPOQ, adiponectin; $T A G$, triacylglycerides; $L D L-C h o$, low-density lipoprotein cholesterol; HDL-Cho, high-density lipoprotein cholesterol; n.a., not applicable

\begin{tabular}{llllll}
\hline & Model $\mathbf{1}$ & & & Model $\mathbf{2}$ & \\
\cline { 2 - 3 } \cline { 5 - 6 } & Bstd & $\boldsymbol{p}$ value & & Bstd & $\boldsymbol{p}$ value \\
\hline BMI-SDS & $-\mathbf{0 . 3 4}$ & $\mathbf{0 . 0 0 8}$ & & n.a. & n.a. \\
Waist circ & $-\mathbf{0 . 3 6}$ & $\mathbf{0 . 0 0 1}$ & & -0.16 & 0.053 \\
TNFa-R1 & -0.14 & 0.313 & & -0.01 & 0.926 \\
log CRP & -0.14 & 0.324 & & 0.03 & 0.856 \\
log ADIPOQ & $\mathbf{0 . 5 8}$ & $<\mathbf{0 . 0 0 1}$ & $\mathbf{0 . 5 7}$ & $<\mathbf{0 . 0 0 1}$ \\
Glucose & -0.19 & 0.198 & & -0.27 & 0.081 \\
Insulin & $-\mathbf{0 . 6 4}$ & $<\mathbf{0 . 0 0 1}$ & $-\mathbf{0 . 6 2}$ & $<\mathbf{0 . 0 0 1}$ \\
HbA1C & -0.03 & 0.823 & & 0.03 & 0.840 \\
HOMA-IR & $-\mathbf{0 . 6 3}$ & $<\mathbf{0 . 0 0 1}$ & $-\mathbf{0 . 6 2}$ & $<\mathbf{0 . 0 0 1}$ \\
log IGI & $-\mathbf{0 . 5 8}$ & $<\mathbf{0 . 0 0 1}$ & $-\mathbf{0 . 5 3}$ & $\mathbf{0 . 0 0 1}$ \\
log WBISI & $\mathbf{0 . 5 9}$ & $<\mathbf{0 . 0 0 1}$ & $\mathbf{0 . 5 7}$ & $<\mathbf{0 . 0 0 1}$ \\
TAG & $-\mathbf{0 . 3 3}$ & $\mathbf{0 . 0 2 8}$ & $-\mathbf{0 . 3 6}$ & $\mathbf{0 . 0 2 5}$ \\
Cholesterol & 0.26 & 0.090 & 0.12 & 0.427 \\
LDL-Cho & 0.27 & 0.076 & 0.14 & 0.363 \\
HDL-Cho & 0.24 & 0.114 & 0.22 & 0.177 \\
\hline
\end{tabular}

insulin levels has been previously described [28, 29], which we also found in our study. However, insulin as a major factor downregulating SHBG production does not come along with the fact that obese subjects are characterized by insulin resistance [30,31], and, to the best of our knowledge, the molecular mechanism by which insulin reduces SHBG production has not yet been described. In this regard, we have demonstrated that insulin does not regulate hepatic SHBG production [15, 32]. Nevertheless, we found strong correlations between SHBG and insulin plasma levels when analyzing our study population or children with obesity alone. Furthermore, SHBG was strongly associated with both insulin sensitivity (HOMA-IR and WBISI) and insulin secretory (IGI) indexes in children with obesity.

Accumulated evidence over the last decade points to inflammation as one of the critical processes associated with the development of obesity, insulin resistance, and type 2 diabetes [33, 34]. In fact, obesity is considered a state of chronic low-grade inflammation [35, 36]. This is of importance since we have reported that alterations in proinflammatory and anti-inflammatory cytokines occurring in obesity $[37,38]$ play an important role in 
regulating hepatic SHBG production [12, 15-17, 39]. Specifically, using in vitro and in vivo models as well as human samples, we have shown that hepatic SHBG production was reduced by TNF $\alpha$ and increased by adiponectin [15-17]. Mechanistically, we have determined that TNF $\alpha$ decreases SHBG production by reducing the protein levels of hepatocyte nuclear receptor 4 alpha $(\mathrm{HNF}-4 \alpha)$, the main activator of $S H B G$ gene expression $[15,16]$. On the other hand, adiponectin increases SHBG production via HNF- $4 \alpha$ levels by inhibiting hepatic lipogenesis and stimulating hepatic $\beta$-oxidation [17]. Interestingly, we showed that plasma TNF $\alpha-R 1$ levels correlated inversely with plasma SHBG levels in adult obese subjects [15]. Remarkably, prepubertal children with obesity from our study had higher TNF $\alpha$-R1 plasma levels than lean children, as described previously [40]. Importantly, SHBG plasma levels correlated significantly and negatively with TNF $\alpha-R 1$ plasma levels. However, this correlation was lost after adjusting for BMI-SDS. This is of importance since TNF $\alpha$ levels could be an important factor downregulating SHBG production during early stages of obesity development, but once children reach a certain point of inflammation, TNF $\alpha$ plasma levels may not influence SHBG production.

Plasma adiponectin levels are lower in subjects with obesity, both adult [41] and children, compared to normal-weight controls [42]. These findings were corroborated in our study, since prepubertal children with obesity had lower levels of adiponectin when compared with lean subjects. In addition, it has been previously reported that adiponectin levels were inversely associated with SHBG [43, 44], and this correlation was also found in our prepubertal population. Moreover, this correlation between SHBG and adiponectin was maintained after adjusting for BMI-SDS. More importantly, this SHBG correlation with adiponectin was also found when analyzing only subjects with obesity.

In conclusion, the results obtained in this study show that prepubertal children with obesity had decreased plasma SHBG levels compared to normal-weight controls. Importantly, SHBG plasma levels correlated significantly with TNF $\alpha$ (negatively) and adiponectin (positively), suggesting that adiponectin and TNF $\alpha$ could play a role in determining plasma SHBG levels in prepubertal children. In addition, our results suggest that plasma adiponectin levels may play a more important role than TNFa in influencing plasma SHBG levels in our prepubertal population with obesity.

\section{Abbreviations}

BMI-SDS: Body mass index standard deviation score:

EDTA: Ethylenediaminetetraacetic acid; ELISA: Enzyme-linked immunosorbent assay; HDL: High-density lipoprotein; HOMA-IR: Homeostasis model

assessment of insulin resistance; LDL: Low-density lipoprotein

\section{Acknowledgements}

We are indebted to the families and children who participated in the study. We thank the "Biobanc de l'Hospital Infantil Sant Joan de Déu per a la Investigació" integrated in the Spanish Biobank Network of Instituto de Salud Carlos III for sample procurement.

\section{Authors' contributions}

MRK: researched data, contributed to discussion, reviewed/edited manuscript. MJLW: contributed to discussion, reviewed/edited manuscript. OOC: contributed to discussion, reviewed/edited manuscript. MAB: contributed to discussion, reviewed/edited manuscript. CL: researched data, contributed to discussion, wrote manuscript, reviewed/edited manuscript.

DMS: researched data, contributed to discussion, wrote manuscript, reviewed/edited manuscript. The authors read and approved the final manuscript

\section{Funding}

This work was supported by grants from the Instituto de Salud Carlos III (DMS) PI12/01357, CIBERDEM (CIBER de Diabetes y Enfermedades Metabólicas Asociadas) an initiative of Instituto de Salud Carlos III (DMS), European Regional Development Funds (ERDF), and Marie Curie FP7-PEOPLE2011-CIG (CL)

\section{Availability of data and materials}

The datasets used and analyzed during the current study are available from the corresponding author on reasonable request.

\section{Declarations}

Ethics approval and consent to participate

All parents signed the informed consent document. The study was approved by the Hospital's Ethic Committee (PIC-21-12).

\section{Consent for publication}

Not applicable.

\section{Competing interests}

The authors declare that they have no competing interests.

Received: 25 September 2020 Accepted: 1 March 2021

Published online: 10 March 2021

\section{References}

1. Wang Y, Lim H (2012) The global childhood obesity epidemic and the association between socio-economic status and childhood obesity. Int Rev Psychiatry 24(3):176-188

2. Fruhstorfer $\mathrm{BH}$, Mousoulis C, Uthman OA et al (2016) Socio-economic status and overweight or obesity among school-age children in sub-Saharan Africa - a systematic review. Clin Obes 6(1):19-32

3. The NS, Suchindran C, North KE et al (2010) Association of adolescent obesity with risk of severe obesity in adulthood. JAMA 304(18):2042-2047

4. Pi-Sunyer $X$ (2009) The medical risks of obesity. Postgrad Med 121(6):21-33

5. Biro FM, Wien M (2010) Childhood obesity and adult morbidities. Am J Clin Nutr 91(5):1499S-1505S

6. Harrell JS, Jessup A, Greene N (2006) Changing our future: obesity and the metabolic syndrome in children and adolescents. J Cardiovasc Nurs 21(4): 322-330

7. Bjerregaard LG, Jensen BW, Ängquist L et al (2018) Change in overweight from childhood to early adulthood and risk of type 2 diabetes. New England Journal of Medicine 378:1302-1312

8. Juonala M, Magnussen CG, Berenson GS et al (2011) Childhood adiposity, adult adiposity, and cardiovascular risk factors. N Engl J Med 365:1876-1885

9. De Moor P, Joossens JV (1970) An inverse relation between body weight and the activity of the steroid binding-globulin in human plasma. Steroidologia 1(3):129-136

10. Kopelman PG, Pilkington TR, White $\mathrm{N}$ et al (1980) Abnormal sex steroid secretion and binding in massively obese women. Clin Endocrinol (Oxf) 12(4):363-369

11. Siiteri PK, Murai JT, Hammond GL et al (1982) The serum transport of steroid hormones. Recent Prog Horm Res 38:457-510 
12. Simó R, Sáez-López C, Barbosa-Desongles A et al (2015) Novel insights in SHBG regulation and clinical implications. Trends Endocrinol Metab 26(7): 376-383

13. Katsuki A, Sumida Y, Murashima S et al (1998) Serum levels of tumor necrosis factor-alpha are increased in obese patients with noninsulindependent diabetes mellitus. J Clin Endocrinol Metab 83(3):859-862

14. Weyer C, Funahashi T, Tanaka S et al (2001) Hypoadiponectinemia in obesity and type 2 diabetes: close association with insulin resistance and hyperinsulinemia. J Clin Endocrinol Metab 86(5):1930-1935

15. Simo R, Barbosa-Desongles A, Lecube A et al (2012) Potential role of tumor necrosis factor-alpha in downregulating sex hormone-binding globulin. Diabetes. 61:372-382

16. Simo R, Barbosa-Desongles A, Sáez-Lopez C et al (2012) Molecular mechanism of TNFalpha-induced down-regulation of SHBG expression. Mol Endocrinol 26:438-446

17. Simó R, Saez-Lopez C, Lecube A et al (2014) Adiponectin upregulates SHBG production: molecular mechanisms and potential implications. Endocrinology 155(8):2820-2830

18. Leal-Witt MJ, Ramon-Krauel M, Samino S et al (2018) Untargeted metabolomics identifies a plasma sphingolipid-related signature associated with lifestyle intervention in prepubertal children with obesity. Int J Obes 42:72-78

19. Leal-Witt MJ, Llobet M, Samino S et al (2018) Lifestyle intervention decreases urine trimethylamine $\mathrm{N}$-oxide levels in prepubertal children with obesity. Obesity (Silver Spring) 26:1603-1610

20. Matsuda M, DeFronzo RA (1999) Insulin sensitivity indices obtained from oral glucose tolerance testing: comparison with the euglycemic insulin clamp. Diabetes Care 22:1462-1470

21. Institute of Medicine (2012) Accelerating progress in obesity prevention: solving the weight of the nation. National Academies Press, Washington DC

22. US Department of Health and Human Services (2010) The surgeon general's vision for a healthy and fit nation. US Dept., Health and Human Services, Rockville, MD

23. Must A, Hollander SA, Economos CD (2006) Childhood obesity: a growing public health concern. Expert Rev Endocrinol Metab 1(2):233-254

24. Adair LS (2008) Child and adolescent obesity: epidemiology and developmental perspectives. Physiol Behav. 22;94(1):8-16.

25. Hammond GL (2011) Diverse roles for sex hormone-binding globulin in reproduction. Biol Reprod 85:431-441

26. Plymate SR, Matej LA, Jones RE et al (1988) Inhibition of sex hormonebinding globulin production in the human hepatoma (Hep G2) cell line by insulin and prolactin. J. Clin. Endocrinol. Metab 67:460-464

27. Loukovaara M, Carson M, Adlercreutz H (1995) Regulation of production and secretion of sex hormone binding globulin in HepG2 cell cultures by hormones and growth factors. J. Clin. Endocrinol. Metab 80:160-164

28. Haffner SM, Katz MS, Stern MP et al (1988) The relationship of sex hormones to hyperinsulinemia and hyperglycemia. Metabolism 37:683-688

29. Pugeat $M$, Cousin P, Baret $C$ et al (2000) Sex hormone-binding globulin during puberty in normal and hyperandrogenic girls. J. Pediatr. Endocrinol. Metab 13:1277-1279

30. Bonadonna RC, Groop L, Kraemer N et al (1990) Obesity and insulin resistance in humans: a dose-response study. Metabolism 39(5):452-459

31. Dunaif A, Segal KR, Futterweit W et al (1989) Profound peripheral insulin resistance, independent of obesity, in polycystic ovary syndrome. Diabetes 38(9):1165-1174

32. Selva DM, Hogeveen KN, Innis SM et al (2007) Monosaccharide-induced lipogenesis regulates the human hepatic sex hormone-binding globulin gene. J Clin Invest 117:3979-3987

33. Wellen KE, Hotamisligil GS (2005) Inflammation, stress, and diabetes. J Clin Invest 115(5):1111-1119

34. Olefsky JM, Glass CK (2010) Macrophages, inflammation, and insulin resistance. Annu Rev Physiol 72:219-246

35. Mathieu P, Lemieux I, Després JP (2010) Obesity, inflammation, and cardiovascular risk. Clin Pharmacol Ther 87(4):407-416

36. Hotamisligil GS (2003) Inflammatory pathways and insulin action. Int J Obes 27:S53-S55

37. Yudkin JS (2003) Adipose tissue, insulin action and vascular disease: inflammatory signals. Int J Obes 27(3):S25-S28
38. Pirola L, Ferraz JC (2017) Role of pro- and anti-inflammatory phenomena in the physiopathology of type 2 diabetes and obesity. World J Biol Chem 26; 8(2):120-128.

39. Simó R, Barbosa-Desongles A, Hernandez C et al (2012) IL1 $\beta$ downregulation of sex hormone-binding globulin production by decreasing HNF4a via MEK-1/2 and JNK MAPK pathways. Mol Endocrinol 26(11):1917-1927

40. Dzienis-Straczkowska S, Straczkowski M, Szelachowska M et al (2003) Soluble tumor necrosis factor-alpha receptors in young obese subjects with normal and impaired glucose tolerance. Diabetes Care 26(3):875-880

41. Oh KW, Lee WY, Rhee EJ et al (2005) The relationship between serum resistin, leptin, adiponectin, ghrelin levels and bone mineral density in middle-aged men. Clin Endocrinol (Oxf) 63:131-138

42. Weiss R, Dufour S, Groszmann A et al (2003) Low adiponectin levels in adolescent obesity: a marker of increased intramyocellular lipid accumulation. J Clin Endocrinol Metab 88(5):2014-2018

43. Vanbillemont G, Lapauw B, De Naeyer H et al (2012) Sex hormone-binding globulin at the crossroad of body composition, somatotropic axis and insulin/glucose homeostasis in young healthy men. Clin Endocrinol (Oxf) 76: $111-118$

44. Yasui T, Tomita J, Miyatani Y, et al (2007) Associations of adiponectin with sex hormone-binding globulin levels in aging male and female populations. Clin Chim Acta 386:69-75.

\section{Publisher's Note}

Springer Nature remains neutral with regard to jurisdictional claims in published maps and institutional affiliations.

\section{Submit your manuscript to a SpringerOpen ${ }^{\circ}$ journal and benefit from:}

- Convenient online submission

- Rigorous peer review

- Open access: articles freely available online

- High visibility within the field

Retaining the copyright to your article

Submit your next manuscript at $\boldsymbol{\nabla}$ springeropen.com 